\title{
Spectrofluorimetry as an Analytical Tool
}

\section{Alok Nahata*}

Department of Pharmaceutical Sciences, Doctor Hari Singh Gour Vishwavidyalaya, SAGAR - 470003 (M.P.) INDIA

Although some information about molecular structure may be derived from excitation and emission spectra, qualitative application of spectrofluorimetry are rare and vast majority of applications in pharmaceutical analysis concern the quantitative assay of drugs, decomposition products and metabolites. Still the use of spectrofluorimetry as an analytical tool provides a well defined identity of the compounds present in the sample on the basis of their unique fluorescent nature

\section{Fluorescence}

When an illuminating system emits light of wavelength different from the incident light, the phenomenon is termed as fluorescence and it takes place as soon as the light is absorbed and ceases as soon as the light is stopped. Fluorescence usually is seen at moderate temperature in liquid solution.

The intensity of the photoluminescence spectrum depends on the excitation wavelength, although its spectral position does not. The photoluminescence spectrum appears at longer wavelengths than the excitation spectrum. This phenomenon arises because the excitation process requires an amount of energy equal to electronic energy change plus a vibrational energy increase; conversely each deexcitation yields the electronic excitation energy minus a vibrational energy increase [1].

\section{High sensitivity and high specificity}

Fluorescence spectroscopy has assumed a major role in analysis, particularly in the determination of trace contaminants in our environment, industries, and bodies, because for applicable compounds fluorescence gives high sensitivity and high specificity. High sensitivity results from a difference in wavelength between the exciting and fluorescence radiation. These results in a signal contrasted with essentially zero background. High specificity results from dependence on two spectra: the excitation and the emission spectrum.

In analytical work, fluorescence is important because of the fact that the intensity of light emitted by a fluorescent material depends upon the concentration of that material. In this manner, the measurement of fluorescence intensity permits the quantitative determination of traces of many inorganic species.

In florescence analysis, the amount of light emitted characteristically under suitable excitation is used as a measure of the concentration of the responsible species.

\section{Factors affecting fluorescence}

The intensity of exciting light is proportional to both the intensity of exciting light and the concentration of the fluorescing material at low concentration $\left(10^{-4}-10^{-7} \mathrm{M}\right)$ and with in narrow limits.

\section{Structural factors:}

- Substituents: Substituents strongly affect fluorescence. A substituent that delocalizes electrons, such as $-\mathrm{NH}_{2}-\mathrm{OH}$, $-\mathrm{F},-\mathrm{OCH}_{3},-\mathrm{NHCH}_{3}$ and $\mathrm{N}\left(\mathrm{CH}_{3}\right)_{2}$ groups, often enhances fluorescence because they tend to increase the transition probability between the lowest excited singlet state and the ground state. Electron withdrawing groups containing $-\mathrm{Cl}$, $-\mathrm{Br},-\mathrm{I},-\mathrm{NHCOCH}_{3},-\mathrm{NO}_{2}$ or $-\mathrm{COOH}$ decrease or quench completely the fluorescence.

- Molecular rigidity: Fluorescence is particularly favoured in molecules that possess rigid structures. Molecular rigidity lessens the possibility of competing nonradiative transitions by decreasing vibrations; this minimizes intersystem crossing to the triplet state and collisional heat degradation. For example, fluorescein and eosin are strongly fluorescent, but a similar compound phenolphthalein, which is non rigid and where the conjugate system is disrupted, is not fluorescent.

- Polarity of the solvent: The polarity of the solvent also affects the fluorescence and phosphorescence. Solvents containing heavy atoms or other such atoms in their structures decrease the fluorescence.

- Presence of dissolved oxygen: The presence of dissolved oxygen often reduces the emission intensity of a fluorescent solution probably due to photochemically induced oxidation of the fluorescent material. Quenching also occurs as a result of paramagnetic properties of molecular oxygen.

- Changes in pH: $\mathrm{pH}$ also exhibits a marked effect on the fluorescence of compounds. For example aniline shows a blue fluorescence in the range $\mathrm{pH} 5-13$, when excited at 290 $\mathrm{nm}$. At lower $\mathrm{pH}$, aniline exists as the aniline cation and in highly alkaline media as the anion. Neither anion nor cation is fluorescent. Fluorescence is more commonly associated with $\pi-\pi *$ states than with $\mathrm{n}-\pi *$ states because $\pi-\pi *$ states possess shorter average lifetime and because deactivation process that compete with fluorescence are much less likely to take place.

- Quenching: Quenching is the reduction in the intensity of fluorescence due to specific effect of constituents of the solution itself. Quenching may be caused in several ways. For example, concentration quenching may be caused by excessive absorption of either primary or fluorescent radiation by the solution. This is also called the inner filter effect. If this effect occurs as a result of absorption by the fluorescent substance itself, the phenomenon is called self-quenching [1,2].

*Corresponding author: Alok Nahata, M.PHARM., Ph.D., Department of Pharmaceutical Sciences, Doctor Hari Singh Gour Vishwavidyalaya, SAGAR - 470003 (M.P.) INDIA, Tel: +919827544352; E-mail: aloknahata@gmail.com

Received September 30, 2011; Accepted October 01, 2011; Published October 03, 2011

Citation: Nahata A (2011) Spectrofluorimetry as an Analytical Tool. Pharm Anal Acta 2:107e. doi:10.4172/2153-2435.1000107e

Copyright: (c) 2011 Nahata A. This is an open-access article distributed under the terms of the Creative Commons Attribution License, which permits unrestricted use, distribution, and reproduction in any medium, provided the original author and source are credited. 


\section{Internal conversion}

The term is used to describe intermolecular processes by which a molecule converts to lower energy electronic state without emission of radiation. Internal conversion is very efficient when two electronic energy levels are sufficient close for an overlap of vibrational levels to exist. The internal conversion through overlapping vibrational levels is usually more probable than the loss of energy by fluorescence from a higher exited state [3].

\section{Relation between intensity of fluorescence and concentration}

The use of fluorescence in quantitative analysis is based on the fact that there should be a definite relationship (preferably linear) between concentration and intensity of fluorescence. On the basis of theory as well as experiment such a linear relationship has actually been found to exist and it is related to the familiar Lambert-beer law, from which it can be derived as under:

The intensity of light absorbed by a solution is $\left(\mathrm{I}_{0}-\mathrm{I}\right)$

Where $\quad I=I_{0} \mathrm{e}^{- \text {acl }}$

Hence the intensity of light absorbed is

$$
\mathrm{I}_{0}-\mathrm{I}_{0} \mathrm{e}^{\text {-acl }} \text { or } \mathrm{I}_{0}\left(\mathrm{I}-\mathrm{e}^{\text {-acl }}\right)
$$

Where

$$
\begin{aligned}
& I_{0}=\text { Intensity of incident light } \\
& I=\text { Intensity of transmitted light }
\end{aligned}
$$

$\mathrm{a}=$ Absorptivity (or extinction coefficient) multiplied by 2.303 .

$$
\begin{aligned}
& c=\text { Concentration } \\
& \mathrm{l}=\text { length of optical path } .
\end{aligned}
$$

The intensity of fluorescence (F) emitted can be obtained by multiplying the amount of light absorbed by quantum yield $(\varphi)$, where $\varphi$ is the ratio of light emitted to the light absorbed. Thus

$$
\mathrm{F}=\varphi \mathrm{XI}_{0}\left(\mathrm{I}-\mathrm{e}^{-\mathrm{acl}}\right)
$$

Reabsorption and scattering of light have not been taken into consideration in the above equation.

It is also assumed that both absorption and emission occur only due to single molecular species and degree of dissociation does not vary with concentration. Now $\mathrm{e}^{\text {-all }}$ can be exponentially expressed as

$$
\mathrm{e}^{-\mathrm{acl}}=1-\mathrm{acl}+\frac{(\mathrm{acl})^{2}}{2}-\frac{(\mathrm{acl})^{3}}{6}+\ldots \ldots \ldots+\frac{(\mathrm{acl})^{\mathrm{n}}}{\mathrm{n} !}
$$

If the magnitude of acl is small, all terms after first two can be neglected. Hence

$$
\mathrm{e}^{-\mathrm{acl}}=1-\mathrm{acl}
$$

Putting in equation (2) we have

$$
\mathrm{F}=\varphi \mathrm{XI}_{0}(1-1+\mathrm{acl})=\varphi \mathrm{XI}_{0} \mathrm{acl}
$$

For a given fluorescent compound, solvent and temperature, and in a cell of definite dimensions, all other factors are constant giving simply,

$$
\mathrm{F}=\mathrm{Kc}
$$

Where $\mathrm{K}$ is proportionality constant.
It is well evident from (4) fluorescent intensity is a linear function of concentration.

\section{Calculation of Results}

It is very important for the analyst to run a blank and two standards of known composition that cover the range of concentration expected. The blank must be kept relatively low and blank reading should be subtracted from all other readings.

$$
\begin{aligned}
\mathrm{F}_{\mathrm{s}} & =\mathrm{KC}_{\mathrm{s}} \\
\mathrm{F}_{\mathrm{u}} & =\mathrm{KC}_{\mathrm{u}}
\end{aligned}
$$

$s$ and $u$ are subscripts for standard and unknown respectively. Standard should be very close to the unknown in composition.

$$
\begin{aligned}
& \text { Dividing (6) by (5) } \\
& \frac{\mathrm{C}_{\mathrm{u}}}{\mathrm{C}_{\mathrm{s}}}=\frac{\mathrm{F}_{\mathrm{u}}}{\mathrm{F}_{\mathrm{s}}} \quad \text { or } \quad \mathrm{Cu}=\frac{\mathrm{C}_{\mathrm{s}} \cdot \mathrm{F}_{\mathrm{u}}}{\mathrm{F}_{\mathrm{s}}}
\end{aligned}
$$

Thus unknown concentration can be determined.

\section{Factors affecting fluorescence intensity}

Fluorescence intensity of a substance is proportional to concentration only when absorbance in a $1 \mathrm{~cm}$ cell is less than 0.02 . If the concentration of fluorescent substance is so great that all incident radiation is absorbed, then

$$
\mathrm{F}=\mathrm{I}_{0} \varphi
$$

i.e. fluorescence is independent of concentration and proportional to the intensity of incident radiation only.

A further problem ensues if the emission and excitation spectra overlap, which results in the reabsorption of fluorescence and a negative dependence of fluorescence on concentration.

\section{Research based on this approach}

We have developed a spectrofluorimetric method to determine coumarins in a well known traditional Indian drug 'Shankhpushpi'. In this study we estimated the amount of total coumarins calculated as scopoletin in different varieties of Shankhpushpi viz., Evolvulus alsinoides and Convolvulus pluricaulis [4]. Further simultaneous estimation of scopoletin and mangiferin in a methanolic extract of Canscora decussata was performed and a successful interpretation about the content of scopoletin and mangiferin in the drug was done [5]. Another attempt in this direction was the development of a spectrofluorimetric method for the determination of curcumin [6]. Recently we have developed a spectrofluorimetric method for the determination of testosterone in biological fluids. The method is specific, precise, accurate and validated [7].

\section{Conclusion}

The method is less tedious and less cumbersome as compared to HPLC and other methods which require long run time and suffer from tedious operation procedures. Moreover the sensitivity offered by this method is far higher than that of HPLC. The compounds can be analysed upto the levels of nanograms. Hence high sensitivity and specificity are real advantages of this method. Further quantitative estimation is possible which can easily measure the amount of the analyte in the mixture. 


\section{References}

1. Willard HH, Merritt LL, Dean JA, Settle FA (1986) Instrumental Methods of Analysis. $6^{\text {th }}$ Edition. CBS Publishers and Distributors, New Delhi. p. 105-111.

2. Beckett $A H$, Stenlake JB (2002) Practical Pharmaceutical Chemistry. $4^{\text {th }}$ Edition Part II. CBS Publishers and Distributors, New Delhi. p. 358-366.

3. Sharma BK (2002) Instrumental Methods of Chemical Analysis. 21 ${ }^{\text {st }}$ Edition Goel Publishing House, Meerut. p. 360-373.

4. Nahata A, Dixit VK (2008) Spectrofluorimetric Estimation of Scopoletin in Evolvulus alsinoides Linn. and Convulvulus pluricaulis Choisy. Indian J Pharm Sci 70: 834-837.
5. Sethiya NK, Nahata A, Dixit VK (2008) Simultaneous Spectrofluorimetric Determination of Scopoletin and Mangiferin in a Methanolic Extract of Canscora decussata Schult. Asian Journal of Traditional Medicines 3: 224-229.

6. Gupta NK, Nahata A, Dixit VK (2010) Development of Spectrofluorimetric Method for the determination of curcumin. Asian Journal of Traditional Medicines 5: 12-18.

7. Nahata A, Gupta NK, Dixit VK (2011) A simple and rapid spectrofluorimetric method for the determination of testosterone in biological fluids. Oriental Pharmacy and Experimental Medicine In Press. 